\title{
Superfície de resposta de medidas morfométricas de Pennisetum glaucum submetidos ao estresse salino
}

\author{
Lucena, L.R.R. ${ }^{\circledR} ;$ Simões, J.V.L.P. e Leite, M.L.M.V.
}

Universidade Federal Rural de Pernambuco. Brasil.

PalaVRas ChaVe ADICIONAIS

Otimização.

Modelagem.

Curva.

Milheto.

Dimensões lineares.

\author{
AdDITIONAL KEYWORDS \\ Optimization. \\ Modelling. \\ Curve. \\ Millet. \\ Linear dimension.
}

\section{INFORMATION}

Cronología del artículo.

Recibido/Received: 30.07 .2018

Aceptado/Accepted: 05.10.2018

On-line: 15.10 .2019

Correspondencia a los autores/Contact e-mail:

leandroricardo_es@yahoo.com.br

\section{RESUMO}

O milheto (Pennisetum glaucum) é uma gramínea de ciclo anual, caracterizada por seu baixo custo de produção, elevada qualidade nutricional e multiplicidade de usos. Objetivou-se encontrar condições ótimas de altura de planta, diâmetro e comprimento de colmo do milheto submetido ao estresse salino através da metodologia de superfície de resposta. Verificou-se que o ponto que maximizou a altura $(192,81 \mathrm{~cm})$ de planta do milheto foi dado pela CEa de 1,8 dS m-1 (salinidade) e uma idade de planta de 45,8 dias. O maior comprimento $(143,7 \mathrm{~cm})$ e diâmetro de colmo $(13,05 \mathrm{~cm})$ do milheto foram definidos pela CEa de 1,8 e 2,47dS m ${ }^{-1}$ e idades de 43,47 e 31,78 dias, respectivamente. A análise de superfície de resposta mostra-se promissora para avaliar a máxima altura de planta, comprimento e diâmetro de colmo do milheto. O grau de salinidade da água de irrigação que maximiza a altura de planta, comprimento e diâmetro do colmo de milheto está situado na faixa entre 1,8 e 2,66 dS m $\mathrm{m}^{-1}$ de condutividade elétrica.

\section{Response surface of morphometric measurements of Pennisetum glaucum submitted to saline stress}

\section{SUMMARY}

The Pearl millet (Pennisetum glaucum) is an annual cycle grass, characterized by its low cost of production, high nutritional quality and multiplicity of uses. The objective was to find optimal conditions of plant height, stem diameter and length of millet submitted to saline stress through the response surface methodology. It was verified that the point that maximized the height $(192.81 \mathrm{~cm})$ of millet plant was given by CEa of $1.8 \mathrm{dS} \mathrm{m} \mathrm{m}^{-1}$ (salinity) and a plant age of 45.8 days. The highest length $(143.7 \mathrm{~cm})$ and stem diameter $(13.05 \mathrm{~cm})$ of millet were defined by CEa of 1.8 and $2.47 \mathrm{dS} \mathrm{m}^{-1}$ and ages of 43.47 and 31.78 days, respectively. Response surface analysis is promising to evaluate the maximum plant height, lenght and stem diameter of millet. The degree of salinity of the irrigation water that maximizes plant height, length and stem diameter of millet its between 1.8 and $2.66 \mathrm{dS} \mathrm{m} \mathrm{m}^{-1}$ of electrical conductivity.

\section{INTRODUÇÃO}

O milheto (Pennisetum glaucum (L.) R. Br.) é uma planta de ciclo anual, e cultivado em vários estados do Brasil, especialmente em lugares em condições de escassez de chuva, fato importante devido sua baixa demanda hídrica (Dan et al. 2010, p. 1118). A importância econômica dessa cultura é caracterizada por seu baixo custo de produção e boa qualidade nutricional (Priesnitz et al. 2011, p. 486), sendo usado na alimen- tação animal, em pastejo ou silagem, como também na produção de grãos para formulação de ração (Pedroso et al. 2009, p. 801).

As medidas morfométricas do milheto como altura de planta, comprimento e diâmetro de colmo são características associadas que influenciam de forma direta na produção de fitomassa, devido aos efeitos importantes que causam no porte físico dos perfilhos (Goes et al. 2011, p. 122). Estas características são positivamente correlacionadas com a produtividade das 
plantas forrageiras (Leite et al. 2017, p. 13). No entanto, vários estádios de desenvolvimento do milheto são afetados pela salinidade da água de irrigação, o que influencia diretamente na produtividade desta planta (Hussain et al. 2010, p. 2108). Elevadas concentrações de sais na solução do solo, principalmente o $\mathrm{NaCl}$, causam a inibição do crescimento das plantas de milheto, em função do decréscimo no potencial hídrico da solução do solo em nível abaixo do necessário para que ocorra a absorção de água pelas células das raízes, e pela toxicidade dos próprios íons (Alves et al. 2011, p. 603). Hussain et al. (2010, p. 2108) verificaram que o aumento de sais no solo devido ao uso de água com baixa qualidade na irrigação causam efeitos danosos no metabolismo dessa cultura.

A metodologia de superfície de resposta (MSR) é uma coleção de técnicas estatísticas úteis para modelagem e análise nas aplicações em que a resposta de interesse seja influenciada por duas ou mais variáveis. O objetivo é otimizar essa resposta, podendo ser representada graficamente e utilizadas de três formas: descrever como as variáveis em teste afetam as respostas, determinar as inter-relações entre as variáveis em teste e descrever efeitos combinados das variáveis em teste sobre a resposta (Achcar et al. 2016, p. 436).

A metodologia de análise de superfície de resposta vem sendo bastante estudada na área de engenharia como relata os trabalhos de Achcar et al. (2016, p. 435); Parka et al. (2012, p. 451); Ramesh et al. (2012, p. 1266). Nas ciências agrárias destacam-se os trabalhos de Shirai e Masson (2010, p. 18) que avaliaram condições de armazenamento de leite cru, Araujo et al. (2011, p. 770) avaliando desempenho de frangos, Chagas et al. (2009, p. 71) avaliando condições ótimas na elaboração de doce de goiaba. Na área de forragilcultura a MSR vem sendo aplicada para verificar os efeitos de diferentes doses de nitrogênio e enxofre nas características produtivas de Brachiaria decumbens (Bonfim-Silva e Monteiro, 2006, p. 1289). Campos et al. (2014, p. 3) utilizando a MSR maximizou a altura de Brachiaria brizantha CV. Xaraés submetidas a diferentes doses de silício e nitro- gênio. Batista e Monteiro (2008, p. 1151) verificaram as características morfogênicas de Brachiaria brizantha cv. Marandu utilizando diferentes doses de nitrogênio e enxofre quando aplicado a MSR.

A superfície de resposta é útil quando o pesquisador não conhece a relação exata entre os fatores. Deste modo, objetivou-se encontrar condições ótimas de altura de planta, diâmetro e comprimento de colmo do milheto submetido ao estresse salino utilizando a metodologia de superfície de resposta.

\section{MATERIAL E MÉTODOS}

A pesquisa foi conduzida de abril a setembro de 2016, na área experimental da Universidade Federal Rural de Pernambuco (UFRPE), Unidade Acadêmica de Serra Talhada (UAST), localizada sob as coordenadas geográficas $07^{\circ} 57^{\prime} 01^{\prime \prime} \mathrm{S}$ e $38^{\circ} 17^{\prime} 53^{\prime \prime} \mathrm{O}$, com altitude aproximada de $523 \mathrm{~m}$, situada no município de Serra Talhada, Microrregião do Sertão do Pajeú, Mesorregião do Sertão Pernambucano (Figura 1).

O clima local é do tipo BSwh', de acordo com a classificação de Koppen, com chuvas de verão, iniciandose em novembro com término em abril, sendo o mês de março historicamente o mais chuvoso. A precipitação pluvial média anual é de $876 \mathrm{~mm}$ e a temperatura do ar média anual é de $27^{\circ} \mathrm{C}$ (Lucena 2016, p. 56).

$\mathrm{O}$ experimento foi instalado em delineamento inteiramente casualizado em quatro níveis de salinidade da água de irrigação, decorrentes de diferentes condutividades elétricas (CEa) $\left(0,1,2\right.$ e $\left.4 \mathrm{dS} \mathrm{m} \mathrm{m}^{-1}\right)$, com quatro repetições, totalizando 16 unidades experimentais. De acordo com a classificação da água para irrigação quanto ao risco de salinidade, proposta pela University of California Commitee of Consultants (Frenkel 1984, p.

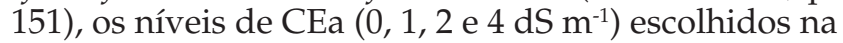
presente pesquisa estão classificados como C1 (baixo risco), C2 (risco médio), e C4 (risco muito alto). Para a obtenção dos níveis salinos foram acrescentados em água destilada sais de cloreto de sódio $(\mathrm{NaCl})$. Em

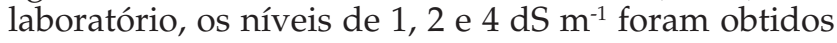

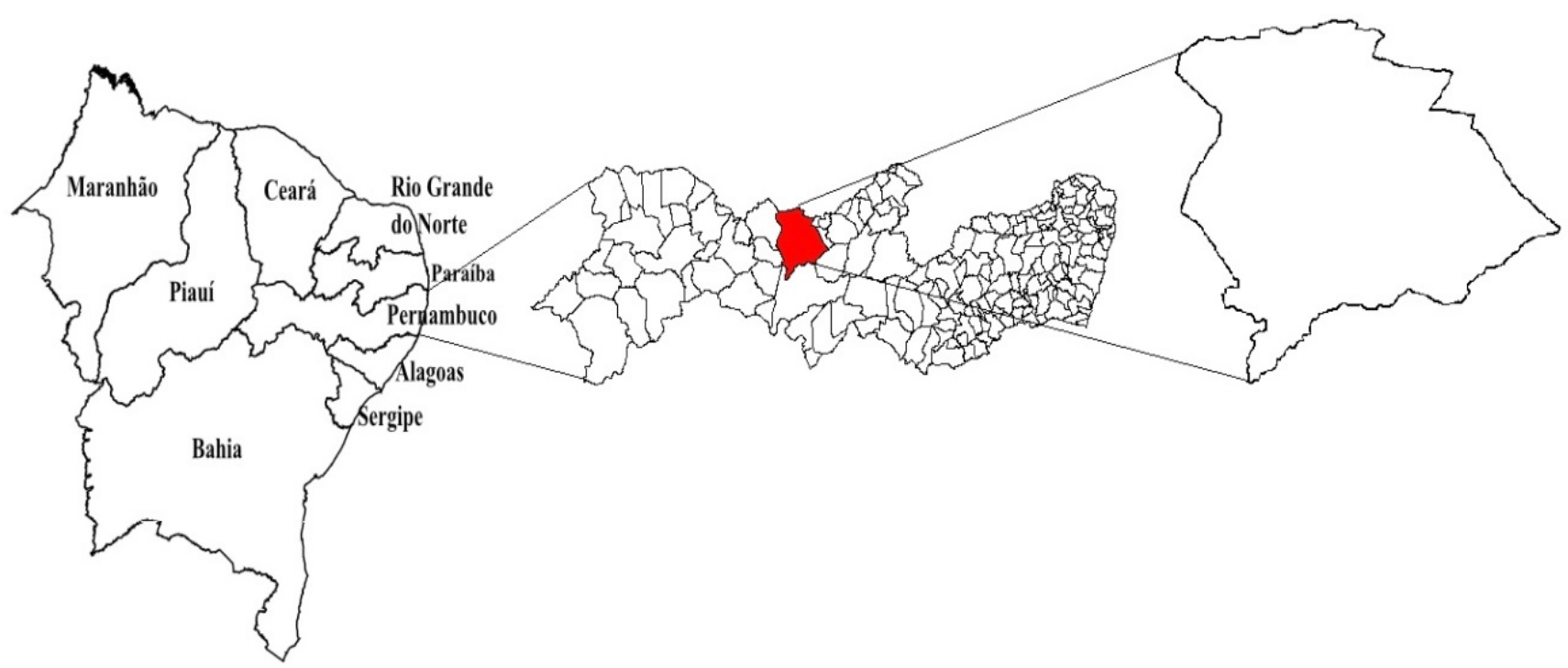

Figura 1. Local de estudo (Lucena, 2016) (Study area (Lucena, 2016) 
através das concentrações de sais de $(\mathrm{NaCl})$ correspondentes à $0,58,1,16$ e $2,33 \mathrm{~g} / \mathrm{L}$, respectivamente. No tratamento com $0 \mathrm{dS} \mathrm{m} \mathrm{m}^{-1}$ (ausência de salinidade), utilizou-se água destilada para irrigação.

Neste ensaio, foram utilizados vasos com 14,41 $\mathrm{dm}^{3}$. Foram dispostos aleatoriamente sobre tijolos, colocando-se $15 \mathrm{~kg}$ de solo. O solo utilizado no experimento, classificado como Cambissolo, conforme descrito por Leite et al. (2017, p. 11), foi coletado na profundidade de $0-20 \mathrm{~cm}$ do perfil, destorroado, homogeneizado, passado em peneira com malha de $2 \mathrm{~mm}$, submetido ao revolvimento para secagem ao ar durante 10 dias e acondicionado em vasos plásticos, perfurados no fundo para drenagem da água de irrigação. Amostra desse solo foi analisada pelo laboratório de fertilidade do solo do Instituto Agronômico de Pernambuco (IPA), caracterizado pelos seguintes atributos químicos: $\mathrm{pH}$ (água) $=7,10 ; \mathrm{P}($ extrator Mehlich $\mathrm{I})=380 \mathrm{mg} \mathrm{dm}^{-3} ; \mathrm{K}^{+}$ $=0,88 ; \mathrm{Ca}^{2+}=1,20 ; \mathrm{Mg}^{2+}=0,10 ; \mathrm{Na}^{+}=0,11 ; \mathrm{Al}^{3+}=0,0$; $\mathrm{H}^{+}=1,00 ; \mathrm{SB}=2,29 ; \mathrm{CTC}=3,29\left(\mathrm{cmol}_{\mathrm{c}} \mathrm{dm}^{-3}\right), \mathrm{m}=0 \%$, $\mathrm{V}=69,60 \%$ e matéria orgânica $=1,24 \%$.

Para a semeadura do milheto, foi usada a cultivar IPA BULK 1BF, colocando-se três sementes por vaso, bem distribuídas, a cerca de $2 \mathrm{~cm}$ de profundidade. O desbaste foi realizado sete dias após a emergência (DAE), procurando-se eliminar as plantas menores e mais fracas, mantendo uma planta por vaso. A irrigação foi realizada com base na evapotranspiração de referência (ETo), sendo determinada através da equação de Penman-Monteith, parametrizada de acordo com o Boletim 56 da FAO (Allen et al. 1998, p. 83).

O tratamento com salinidade começou aos 15 DAE, antes disso as irrigações foram feitas sem a presença de sais na água. Este período foi considerado visando o estabelecimento das plântulas. Semanalmente, foram efetuadas análises biométricas de todas as plantas até os 56 (DAE), objetivando monitorar o crescimento da cultura, através das seguintes variáveis: altura de plantas; comprimento do colmo; diâmetro do colmo. A altura das plantas e o comprimento do colmo foram determinados por meio de uma trena, medindo-se a distância vertical da base do colmo até a extremidade da curvatura da última folha completamente expandida da planta e a distância vertical entre a superfície do solo e o ápice do colmo, respectivamente. Com relação ao diâmetro do colmo, considerou-se a medida a $5 \mathrm{~cm}$ do nível do solo utilizando um paquímetro digital.

Para análise da altura de planta (AP), diâmetro (DC) e comprimento (CC) de colmo do milheto foi utilizada a metodologia de análise de superficie de resposta. Definiu-se a superfície de resposta para este estudo como sendo a representação geométrica obtida quando uma variável resposta é plotada como uma função de dois fatores quantitativos. A função pode ser assim definida:

$$
\mathrm{Y}=\mathrm{f}\left(\mathrm{X}_{1}, \mathrm{X}_{2}\right)+\varepsilon
$$

onde, Y é a resposta (altura de planta, diâmetro de colmo e comprimento de colmo); são os fatores (grau de salinidade e tempo) $\mathrm{X}_{1}$ e $\mathrm{X}_{2}$ é $\varepsilon$ o erro aleatório. Denota-se a resposta esperada por:

$$
\mathrm{E}(\mathrm{Y})=\mathrm{f}\left(\mathrm{X}_{1}, \mathrm{X}_{2}\right)=\eta
$$

$$
\begin{aligned}
& \text { então, } \\
& \eta=\mathrm{f}\left(\mathrm{x}_{1}, \mathrm{x}_{2}\right)
\end{aligned}
$$

é chamada de superficie de resposta (Achcar et al. 2016, p. 440).

No presente estudo a função $\mathrm{f}\left(\mathrm{X}_{1}, \mathrm{X}_{2}\right)$ é definida por:

$$
Y=f\left(X_{1}, X_{2}\right)+\varepsilon=\beta_{0}+\beta_{1} X_{1}+\beta_{2} X_{2}+\beta_{3} X_{1} X_{2}+\beta_{4} X_{1}^{2}+\beta_{5} X_{2}^{2}+\varepsilon
$$

em que $\beta_{0}, \beta_{1}, \beta_{2}, \beta_{3}, \beta_{4}$ e $\beta_{5}$ e $\varepsilon$ são os parâmetros desconhecidos.

$$
\mathrm{x}_{1 \mathrm{u}}=\frac{\mathrm{x}_{1 \mathrm{u}}-\bar{X}_{1}}{s \mathrm{X}_{1}} \text { e } \mathrm{x}_{2 \mathrm{u}}=\frac{\mathrm{x}_{2 \mathrm{u}}-\bar{X}_{2}}{\mathrm{x}_{2}}
$$

onde, $\mathrm{u}=1,2, \ldots, \mathrm{N}$; N é o total de observações e $X_{1 u}$ e $X_{2}$ é o u-ésimo nível das variáveis independentes (grau de salinidade e tempo),

$$
\bar{X}_{1}=\sum_{\mathrm{u}=1}^{\mathrm{N}} \mathrm{X}_{1 \mathrm{u}} / \mathrm{N} \text { e } \bar{X}_{2}=\sum_{\mathrm{u}=1}^{\mathrm{N}} \mathrm{X}_{2 \mathrm{u}} / \mathrm{N}
$$

é a média amostral das variáveis $\mathrm{X}_{1}$ e $\mathrm{X}_{2}$ e

são os desvio padrões de $\mathrm{X}_{1}$ e $\mathrm{X}_{2}$.

Assim a variável resposta $Y$ fica definida depois da codificação das variáveis por:

$$
Y_{u}=\beta_{0}+\beta_{1} x_{1 u}+\beta_{2} x_{2 u}+\beta_{3} x_{1 u} x_{2 u}+\beta_{4} x_{1 u}^{2}+\beta_{5} x_{2 u}^{2}+\varepsilon_{u}
$$

onde, é independente distribuido com média zero e variância constante.

O modelo acima pode ser escrito na seguinte forma matricial:

$$
\mathrm{Y}=\beta \mathrm{X}+\varepsilon
$$

em que, $Y^{\prime}=\left[Y_{1}, Y_{2}, \ldots, Y_{N}\right], X$ é a matriz $\mathrm{N} x \mathrm{p} ; \mathrm{p}=6$; $\beta$ é um vetor $\mathrm{p}$ × 1 de parâmetros desconhecidos $\varepsilon^{\prime}=\left[\varepsilon_{1}, \varepsilon_{2}, \ldots, \varepsilon_{N}\right]$. Utilizando o estimador de mínimos quadrados para o modelo tem-se que a estimativas dos parâmetros é dada por:

$$
\widehat{\beta}=\left(X^{\prime} X\right)^{-1} X^{\prime} Y
$$

A fim de encontrar o grau de salinidade (SAL) e a idade ideal $(\mathrm{T})$ do milheto que maximize a altura da planta, o diâmetro e o comprimento do colmo recorremos ao ponto estacionário. Este ponto se existir será calculado da seguinte forma:

$$
\frac{\partial \hat{\mathrm{Y}}}{\partial \mathrm{x}_{1}}=0 \text { e } \frac{\partial \hat{\mathrm{Y}}}{\partial \mathrm{x}_{2}}=0
$$

ou seja, resolver o seguinte sistema de equação:

$$
\begin{gathered}
\frac{\partial \widehat{\mathrm{Y}}}{\partial \mathrm{X}_{1}}=\hat{\beta}_{1}+2 \hat{\beta}_{3} \mathrm{X}_{1}+\hat{\beta}_{5} \mathrm{X}_{2}=0 \\
\mathrm{e} \\
\frac{\partial \widehat{\mathrm{Y}}}{\partial \mathrm{X}_{2}}=\hat{\beta}_{2}+2 \hat{\beta}_{4} \mathrm{X}_{2}+\hat{\beta}_{5} \mathrm{X}_{1}=0
\end{gathered}
$$

Para testar a significância do modelo ajustado é realizado um procedimento de análise de variância 
(ANOVA). Considerando o sistema de equações normais abaixo temos:

$$
X^{\prime} X \widehat{\beta}=X^{\prime} Y
$$

Podemos obter as somas de quadrados total (SQT), regressão (SQReg) e residuos (SQR) pela ANOVA,

$$
\begin{gathered}
\mathrm{SQT}=\mathrm{Y}^{\prime} \mathrm{Y}-\mathrm{C}=\sum_{\mathrm{i}=1}^{\mathrm{N}} \mathrm{Y}_{\mathrm{i}}^{2}-\mathrm{C} \\
\mathrm{SQR}=\mathrm{Y}^{\prime} \mathrm{Y}-\widehat{\beta}^{\prime} \mathrm{X}^{\prime} \mathrm{Y} \\
\mathrm{SQReg}=\widehat{\beta}^{\prime} \mathrm{X}^{\prime} \mathrm{Y}-\mathrm{Y}^{\prime} \mathrm{Y}
\end{gathered}
$$

em que,

$$
C=\frac{\left(\sum_{i=1}^{N} Y_{i}\right)^{2}}{N}
$$

Para avaliar a adequação do modelo, foi utilizado o roeficiente de determinacão do modelo ajustado $\left(\mathrm{R}_{\mathrm{a}]}^{2} \mathrm{R}_{\mathrm{aju}}^{2}=\frac{\mathrm{R}^{2}(\mathrm{~N}-1)-\mathrm{p}}{\mathrm{N}-\mathrm{p}-1} ; \quad 0 \leq R_{a j u}^{2} \leq 1\right.$.

onde,

$$
R^{2}=\frac{S Q R e g}{S Q T} .
$$

\section{RESULTADOS E DISCUSSÃO}

Na avaliação da altura de planta do milheto o modelo ajustado ficou definido pela seguinte equação:

$$
\mathrm{AP}=-18,13 \mathrm{SAL}+9,7 \mathrm{~T}-0,3 \mathrm{SAL} * \mathrm{~T}+4,84 \mathrm{SAL}^{2}-0,1 \mathrm{~T}^{2}
$$

O modelo ajustado apresentou os parâmetros significativos ( $\mathrm{p}$-valor $<0,0001)$ através da ANOVA, tal modelo apresentou $\mathrm{SQR}=95520$ e grau de explicação $\left(\mathrm{R}_{\text {aju }}^{2}\right)$ de $96,74 \%$, Tabela I.

Na Figura 2, observa-se o comportamento da superficie de resposta para altura de planta do milheto, nota-se que através da superficie de resposta o ponto que maximizou a altura de planta $(192,81 \mathrm{~cm})$ foi dado pela CEa de 1,8 $\mathrm{dS} \mathrm{m} \mathrm{m}^{-1}$ (salinidade) e uma idade de planta de 45,8 dias (DAE).

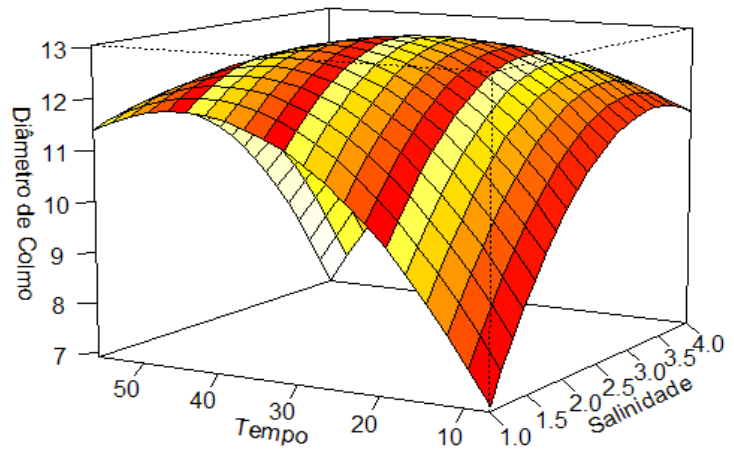

Figura 2. Superfície de resposta para altura de planta de milheto (Response surface for plant height of millet).
Tabela I. Análise de variância do modelo para altura de planta de milheto (Analysis of variance of plant height model of millet).

\begin{tabular}{lccccc}
$\begin{array}{l}\text { Fonte de } \\
\text { Variação }\end{array}$ & gl & SQ & QM & F & p-valor \\
\hline Regressão & 5 & 16196,7 & 3239,24 & 469,47 & $<0,0001$ \\
Resíduo & 123 & 846 & 6,9 & & \\
\hline Total & 128 & 17042,7 & & & \\
\hline
\end{tabular}

A altura de planta é uma característica correlacionada com a produtividade de fitomassa, tornando-se uma variável importante a ser avaliada. Na literatura, observou-se grande variação na altura de plantas de milheto, o que deve ser interpretado de forma cuidadosa, já que ela é muito influenciada pela idade da planta no período de avaliação, condições edafoclimáticas de cultivo, adubação e época de semeadura da cultura (Neumann et al. 2002, p. 303).

A altura de planta encontrada nesta pesquisa foi similar às encontradas na maioria dos trabalhos com milheto (Costa et al. 2013, p. 350; Domukoski et al. 2014, p. 155). O excesso de sais no solo provoca redução do desenvolvimento vegetal, podendo ser seu efeito de forma direta, indireta, lenta ou brusca, total ou parcial. Sais solúveis na solução do solo, principalmente Na e $\mathrm{Cl}$ causam distúrbios fisiológicos, principalmente em espécies mais susceptíveis (Farias et al. 2009, p. 1500). As altas concentrações de sais no solo tornam mais difícil para as raízes extrair água e altas concentrações de sais dentro da planta podem ser tóxicas (Munns e Tester 2008, p. 652).

A curva ajustada em relação ao comprimento de colmo é expressa pela equação:

$$
\mathrm{CC}=-26,91 \mathrm{SAL}+8,26 \mathrm{~T}+5,05 \mathrm{SAL}^{2}-0,095 \mathrm{~T}^{2}
$$

Através do teste da ANOVA verificou-se que o modelo ajustado para o comprimento de colmo do milheto foi significativo ( $p$-valor $<0,0001$ ), Tabela II. O modelo aiustado apresentou $\mathrm{SQR}=78508$ e poder de explicação $\left(\mathrm{R}_{\mathrm{aju}}^{2}\right)$ de $95,80 \%$, indicando adequação no modelo ajustado.

A superficie de resposta para o comprimento de colmo está apresentada na Figura 3. Observou-se que o ponto que maximizou o comprimento de colmo do milheto $(143,70 \mathrm{~cm})$ foi definido pela CEa de 2,66 dS $\mathrm{m}^{-1}$ (salinidade) e idade da planta de 43,47 dias (DAE).

O efeito osmótico da salinidade sobre as plantas é causado devido a elevadas concentrações de sais dissolvidos na solução do solo, provocando uma redução

Tabela II. Análise de variância do modelo para o comprimento de colmo de milheto (Analysis of variance of model for stem length of millet).

\begin{tabular}{lccccc}
\hline $\begin{array}{l}\text { Fonte de } \\
\text { Variação }\end{array}$ & gl & SQ & QM & F & p-valor \\
\hline Regressão & 4 & 1848836 & 462209 & 730,188 & $<0,0001$ \\
Resíduo & 124 & 78508 & 633 & & \\
\hline Total & 128 & 1927344 & & & \\
\hline
\end{tabular}




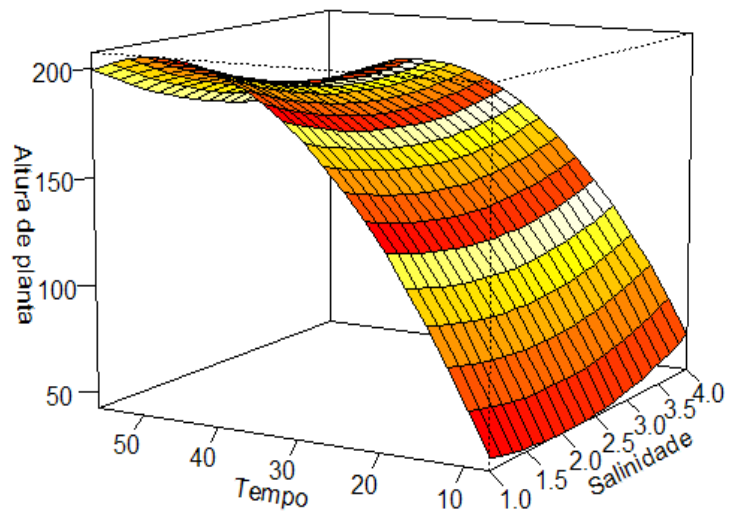

Figura 3. Superfície de resposta do comprimento do colmo de milheto (Response surface of length of stem of millet).

Tabela III. Análise de variância do modelo para o diâmetro de colmo de milheto (Analysis of variance of model for stem diameter of millet).

\begin{tabular}{lccccc}
\hline $\begin{array}{l}\text { Fonte de } \\
\text { Variação }\end{array}$ & Gl & SQ & QM & F & p-valor \\
\hline Regressão & 5 & 2949539 & 589907,8 & 6,176 & $<0,0001$ \\
Resíduo & 123 & 95520 & 777 & & \\
\hline Total & 128 & 3045059 & & & \\
\hline
\end{tabular}

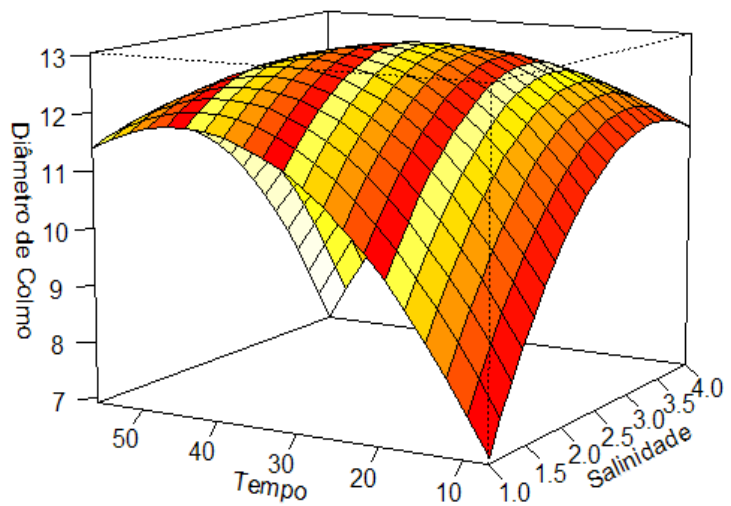

Figura 4. Superfície de resposta do diâmetro de colmo de milheto (Response surface of stem diameter of millet). no seu potencial osmótico, diminuindo a disponibilidade e água de nutrientes. Com o aumento da pressão osmótica poderá ocorrer seca fisiológica e, dependendo do grau de salinidade, a planta em vez de absorver, poderá perder a água que se encontra no interior das raízes (Alves et al. 2011, p. 603; Schossler et al. 2012, p. 1568). Na ocorrência de seca fisiológica a planta interrompe o seu metabolismo e consequentemente o seu crescimento é cessado, o que representa um impacto direto nas variáveis altura e comprimento de colmo e produtividade das plantas de milheto, uma vez que a água que se encontra no interior das raízes é reabsorvida pelo solo.

Na avaliação do diâmetro de colmo de milheto o modelo ajustado ficou definido pela seguinte equação:

$$
\mathrm{DC}=5,81 \mathrm{SAL}+0,37 \mathrm{~T}-0,059 \mathrm{SAL} * \mathrm{~T}-0,79 \mathrm{SAL}^{2}-0,0035 \mathrm{~T}^{2}
$$

$\mathrm{O}$ modelo supracitado apresentou significância através da ANOVA, (Tabela III). Tal modelo apresentou $S Q R=846,0$ e grau de explicação $\left(R_{\text {aju }}^{2}\right)$ de $94,83 \%$ - Assim a curva ajustada apresentou adequação para explicar o diâmetro do colmo.

$\mathrm{Na}$ Figura 4 verifica-se o comportamento da curva ajustada do diâmetro de colmo de milheto em função da idade da planta e do grau de salinidade avaliado.

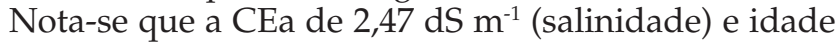
de planta de 31,78 dias (DAE), promoveram o maior diâmetro de colmo $(13,05 \mathrm{~mm})$.

O diâmetro de colmo é uma variável que influencia de forma direta a produção de fitomassa, devido aos efeitos importantes que essa característica causa no porte físico dos perfilhos (Goes et al. 2011, p. 125). Oliveira et al. (2015, p. 151) ressaltaram que o diâmetro do colmo do milho pipoca foi incrementado com o aumento da salinidade da água de irrigação até 2,13 $\mathrm{dS} . \mathrm{m}^{-1}$, a partir deste ponto o diâmetro do colmo foi reduzido. Pinho et al. (2013, p. 430) notaram valores médios de diâmetro de colmo de milheto variando entre 0,59 a $0,94 \mathrm{~cm}$.

Nas espécies glicófitas, como por exemplo o milheto, a tolerância à salinidade está relacionada com à habilidade da planta em evitar a acumulação de íons tóxicos na parte aérea, principalmente, nas folhas. No entanto, as espécies sensíveis não conseguem evitar esse acúmulo e nem realizar uma compartimentalização desses íons em tecido específico, ocasionando na interferência dos mesmos no metabolismo celular, e consequentemente na redução no crescimento e desenvolvimento (Boursier e Lauchli 1989, p. 543).

De acordo com Hussain et al. (2008, p. 25) no estudo com duas cultivares de milheto, o conteúdo mais alto dos íons de $\mathrm{Na}^{+} \mathrm{e} \mathrm{Cl}^{-}$foram encontrados por ordem em colmos e bainhas, raízes, folhas jovens ( $2^{\mathrm{a}}$ folha, a partir do topo) e folhas antigas ( $3^{\mathrm{a}}$ folha, a partir da parte superior). Esses resultados de retenção de íons potencialmente tóxicos no colmo e bainha são considerados como um exemplo de um mecanismo de tolerância do milheto à salinidade, sugerindo que esta espécie exporta pouco $\mathrm{Na}^{+}$do caule para os limbos foliares, evitando, assim, o excesso de íons potencialmente tóxicos nos tecidos fotossintetizantes. 


\section{CONCLUSÕES}

Os processos de crescimento são particularmente sensíveis aos efeitos dos sais, de modo que as características morfométricas avaliadas podem constituir um bom critério para a avaliação do grau de estresse e da capacidade adaptativa do milheto ao estresse salino.

A análise de superfície de resposta mostra-se promissora para avaliar características morfométricas do milheto. O grau de salinidade da água de irrigação que maximiza a altura de planta, comprimento e diâmetro do colmo de milheto está situado na faixa entre 1,8 e 2,66 dS m $\mathrm{m}^{-1}$ de condutividade elétrica.

\section{BIBLIOGRAFIA}

Achcar, JA, Faria, RF, \& Piratelli, CL 2016, 'Metodologia de superfícies de respostas aplicadas em usinagem de metais', Revista Brasileira de Biometria, vol. 34, no. 3, pp. 435-54

Allen, RG, Pereira, LS, Raes, D, \& Smith, M 1998, 'Crop evapotranspiration: guidelines for computing crop water requirements', Irrigation and drainage, vol. 56, no. 1, pp. 1-300.

Alves, FAL, Silva, SLF, Silveira, JAG, \& Pereira, VLA 2011 , 'Efeito do Ca2+ externo no conteúdo de $\mathrm{Na}+\mathrm{e} \mathrm{K}+$ em cajueiros expostos a salinidade', Revista Brasileira de Ciências Agrárias, vol. 6, no. 4, pp. 602-08.

Araujo, RB, Faria, DE, Faria Filho, DE, Lima, CG, Trevisan, RB, Souza, KMR, Sakamoto, MI, \& Souza, VN 2011, 'Modelos de superfície de resposta para predição do desempenho de frangos e elaboração de análise económica', Revista Brasileira de Saúde e Produção Animal, vol. 12, no. 3, pp. 770-83.

Batista, K \& Monteiro, FA 2008, 'Nitrogênio e enxofre nas características morfogênicas do capim-marandu em substituição ao capim-braquiária em degradação em solo com baixo teor de matéria orgânica', Revista Brasileira de Zootecnia, vol. 37, no. 7, pp. 1151-1160.

Bonfim-Silva, EM \& Monteiro, FA 2006, 'Nitrogênio e enxofre em características produtivas do capim-braquiária proveniente de área de pastagem em degradação', Revista Brasileira de Zootecnia, vol. 35, no. 4, pp. 1289-1297.

Bousier, P \& Lauchli, A 1989, 'Mechanisms of chloride partitioning in the leaves of salt stressed Sorghum bicolor L.', Physiology Plant, vol. 77, no. 4, pp. 537-544

Campos, OHG, Lizzi, JB, Santos, MP, \& Melo, SP 2014, 'Silício e nitrogênio no estabelecimento da Brachiaria brizantha cv. Xaraés', Synergismus Scyentifica UTFPR, vol. 9, no. 1, pp. 1-6.

Chagas, EN, Menezes, CC, Cirillo, MA, \& Borges, SV 2009, 'Método "ridge" em modelo de superfície de resposta: otimização de condições experimentais na elaboração de doce de goiaba', Revista Brasileira de Biometria, vol. 26, no. 4, pp. 71-81.

Costa, ACT, Erig, MC, Reginatto, JRR, Domukoski, JF, Ecco, M, Duarte Jr., JB, \& Vasconcelos, E D 2013, 'Comportamento do milheto na safra de verão em função do espaçamento entre linhas e densidade de semeadura', Scientia Agraria Paranaensis, vol. 12, no. 1, pp. 347-53.

Dan, HA, Barroso, ALL, Procópio, SO, Dan, LGM, Finotti, TR, \& Assis, RL 2010, 'Seletividade do atrazine à cultura do milheto (Pennisetum glaucum)', Planta Daninha, vol. 28, no. 4, pp. 1117-24.

Domukoski, JF, Costa, ACT, Lázaro, RL, Silva, KF, Ecco, E, \& Duarte Jr., JB 2014, 'Produção de biomassa de milheto em função do espaçamento entrelinhas e da densidade de semeadura', Scientia Agraria Paranaensis, vol. 13, no. 2, pp. 152-60.

Farias, SGG, Santos, DR, Freire, ALO, \& Silva, RB 2009, 'Estresse salino no crescimento inicial e nutrição mineral de Gliricídia (Gliricidia sepium (Jacq.) Kunt ex Steud) em solução nutritiva', Revista Brasileira de Ciência do Solo, vol. 33, no. 5, pp. 1499-1505.

Frenkel, H 1984, 'Reassessment of water quality criteria for irrigation. In: Shainberg. I, Shalhevet, J', Soil salinity under irrigation, vol. 1, pp. 143-72.
Goes, RJ, Rodrigues, RAF, Arf, O, De Arruda, OG, \& Vilela, RG 2011, 'Fontes e doses de nitrogenio em cobertura, no sorgo granifero na safrinha', Revista Brasileira de Milho e Sorgo, vol. 10, no. 2, pp. 121-9.

Hussain, K, Majeed, A, Nawaz, K, Nisar, FK, Khan, F, Afghan, S, \& Ali, $K$ 2010, 'Comparative study for salt stress among seed, root stock and direct regenerated violet (Viola odorata L.) seedlings in relation to growth, ion contents and enzyme activities', African Journal Biotechnology, vol. 9, no. 14, pp. 2108-17.

Hussain, K, Ashraf, M, \& Ashraf, MY 2008, 'Relationship between growth and ion relation in pearl millet (Pennisetum glaucum (L.) R. $\mathrm{Br}$.) at different growth stages under salt stress', African Journal of Biotechnology, vol. 2, no. 3, pp. 23-27.

Leite, MLMV, Lucena, LRR, SÁ Jr, EH, \& Cruz, MG 2017, 'Estimativa da área foliar em Urochloa mosambicensis por dimensões lineares', Revista Agropecuária Técnica, vol. 38, no. 1, pp. 9-16.

Lucena, LRR 2016, 'Utilização de lacunaridade na caracterização do vento em Serra Talhada-PE', Biomatemática, vol. 26, no. 1, pp. 53-64.

Munns, R \& Tester, M 2008, 'Mechanisms of Salinity Tolerance', Annual Review of Plant Biology, vol. 59, no. 1, pp. 651-81.

Neumann, M, Restle, J, Alves Filho, DC, Bernardes, RAC, Arboite, MZ, Cerdótes, L, \& Peixoto, LAO 2002, 'Avaliação de diferentes híbridos de sorgo (Sorghum bicolor, L. Moench) quanto aos componentes da planta e silagens producidas', Revista Brasileira de Zootecnia, vol. 31, no. 1, pp. 302-12.

Oliveira, FDA, Medeiros, JF, Oliveira, MK, Lima, CJDS, Almeida Jr. , AB, \& Amâncio, MDG 2015, 'Desenvolvimento inicial do milho-pipoca irrigado com água de diferentes níveis de salinidade', Revista Brasileira de Ciências Agrárias (Agrária), vol. 4, no. 2, pp. 149-55.

Parka, SJ, Kimb, DH, \& Leeb, CM 2012, 'A study determining the optimal machining conditions for a 40,000 rpm ultra high speed spindle system', Precision Engineering, vol. 36, no. 1, pp. 451-57.

Pedroso, CES, Monks, PL, Ferreira, OGL, Lima, LS, \& Tavares, OM 2009, 'Características estruturais de milheto sob pastejo rotativo com diferentes períodos de descanso', Revista Brasileira de Zootecnia, vol. 38, no. 5, pp. 801-08.

Pinho, RMA, Santos, EM, Rodrigues, JAS, Macedo, CHO, Campos, FS, Ramos, JPDF, Bezerra, HFC, \& Pezarro, AF 2013, 'Avaliação de genótipos de milheto para silagem no semiárido', Revista Brasileira de Saúde e Produção Animal, vol. 14, no. 3, pp. 426-36.

Priesnitz, R, Costa, ACT, Jandrey, PE, Frez, JRS, Duasrte Jr., JB, \& Oliveira, PSR 2011, 'Espaçamento entre linhas na produtividade de biomassa e de grãos em genótipos de milheto pérola', Semina: Ciências Agrárias, vol. 32, no. 2, pp. 485-94.

Ramesh, S, Karunamoorthy, L, \& Palanikumar, K 2012, 'Measurement and analysis of surface roughness in turning of aerospace titanium alloy (gr5)', Measurement, vol. 45, no. 5, pp. 1266-76.

Schossler, TR, Machado, DM, Zuffo, AM, Andrade, FR, \& Piauilino, AC 2012, 'Salinidade: efeitos na fisiologia e na nutrição mineral de plantas', Enciclopédia Biosfera, vol. 8, no. 15, pp. 1563-78.

Shirai, MA \& Masson, ML 2010, 'Metodologia de superfície de resposta para avaliar o efeito da carbonatação sobre a microbiota e viscosidade do leite cru', Revista do Instituto de Laticínios Cândido Tostes, vol. 65 , no. 375 , pp. $18-27$. 